\title{
Gestão da qualidade na administração pública: autoavaliação sobre a aplicação de práticas em órgãos do Sistema Nacional de Vigilância Sanitária
}

\section{Quality management in public administration: self-evaluation on the application of practices in the National Health Surveillance System}

\author{
Wilma Madeira da Silva' iD \\ Vera Maria Borralho Bacelar' iD \\ Artur luri Alves de Sousa" (iD) \\ Danila Augusta Accioly Varella \\ Barcal iD
}

Cláudio Medeiros Santos' (iD)

Bruno Lopes Zanetta' i
Hospital Alemão Oswaldo Cruz, São Paulo, SP, Brasil

" Agência Nacional de Vigilância Sanitária (Anvisa), Brasília, DF, Brasil

\section{* E-mail: wilma.madeira@gmail.com}

Recebido: 17 nov 2020 Aprovado: 28 abr 2021

\author{
RESUMO
}

Introdução: A gestão da qualidade é um mecanismo de destaque no contexto gerencial, firmando-se como espaço teórico e prático de produção de conhecimento e influenciando a execução e o controle nos processos de trabalho nas organizações. Objetivo: Identificar a aplicação de práticas de gestão da qualidade, por meio de autoavaliação, em órgãos de Vigilância Sanitária. Método: Estudo exploratório descritivo, de autoavaliação, com uso de formulário eletrônico para a coleta de dados. Foram selecionados e aplicados requisitos considerados iniciais em um modelo de Sistema de Gestão da Qualidade, a partir da norma ABNT NBR ISO 9001:2015. Com o intuito de consultar órgãos de Vigilância Sanitária que exercessem ações envolvendo atividades de alto risco sanitário, foram considerados municípios e estados em cujos territórios houvesse concentração de, pelo menos, três unidades industriais fabricantes de medicamentos, insumos farmacêuticos ativos e produtos para a saúde classes de risco III e IV. Assim, o universo do estudo compreendeu sete estados e 32 municípios, nos quais estavam instalados, respectivamente, $94,0 \%$ e $63,0 \%$ do parque industrial brasileiro das referidas indústrias, no ano de 2018. Resultados: Observou-se que, nos órgãos de Vigilância Sanitária estudados, existem práticas de gestão da qualidade sendo executadas, sendo que as práticas de planejamento e apoio são as mais difundidas, enquanto as voltadas à avaliação de desempenho são as menos presentes. Conclusões: Conhecer o grau em que os órgãos de Vigilância Sanitária atendem aos requisitos iniciais de cada uma das seções de um modelo de Sistema de Gestão da Qualidade contribui para confirmar o potencial para implantação de princípios da Gestão da Qualidade nos entes que compõem o Sistema Nacional de Vigilância Sanitária, para que esses estejam em sintonia com as exigências de melhoria de gestão impostas pelo ambiente regulatório nacional e internacional.

PALAVRAS-CHAVE: Gestão da Qualidade; Vigilância Sanitária; ISO 9001

\section{ABSTRACT}

Introduction: Quality management is an outstanding mechanism in the managerial context, established as a theoretical and practical space of knowledge production, influencing the execution and control of processes in organizations. Objective: Identifying by means of self-evaluation the application of quality management practices in Health Surveillance Agencies. Method: Exploratory, descriptive, self-evaluation study using an electronic form for data collection, regarding a set of requirements deemed as initial stage of the Quality Management System model based on the ABNT NBR ISO 9001: 2015 Standard. In order to consult with Health Surveillance Agencies that deal with activities of high sanitary risk, municipalities and states in whose territories there were at least three factories of either medicines or active pharmaceutical ingredients or healthcare products classes of risk III and IV were selected. Thus, the universe of study comprehends seven states and 32 municipalities, with $94.0 \%$ and $63.0 \%$ of the Brazilian industrial park 
for these kinds of industry, in 2018. Results: In every agency there are quality management practices being performed: practices related to Planning and Support are the most widespread; those relative to Performance Evaluation are the least present. Conclusions: Grasping the degree to which Health Surveillance Agencies comply with the starting set of requirements of each section of the Model has contributed to confirm the potential of implementing the principles of the Quality Management System in the entities of the National Health Surveillance System, so they are in line with the demands for management improvement imposed by the national and international regulatory environment.

KEYWORDS: Quality Management; Health Surveillance; ISO 9001

\section{INTRODUÇÃOO}

A gestão da qualidade (GQ) tem ocupado posição de destaque no contexto gerencial, firmando-se como espaço teórico e prático de produção de conhecimento, influenciando a execução e o controle do trabalho nas organizações ${ }^{1}$. Fenômeno mundial desde a década de 1980, observa-se que muitas e diferentes organizações têm investido esforços na qualificação de seus processos de trabalho, por meio da incorporação de práticas oriundas de Sistemas de Gestão da Qualidade (SGQ) ${ }^{2}$.

Como tema de crescente importância, cada vez mais organizações em todo o mundo têm implantado SGQ usando como referencial normativo a ISO 9001 que, elaborada pela International Organization for Standardization (ISO), foi desenvolvida para atender organizações que buscam melhorar a qualidade de seus processos, produtos e serviços. A ABNT NBR ISO 9001, norma que estabelece o conjunto de requisitos para um SGQ, pode ser aplicada a qualquer ramo de atividade e tem por finalidade proporcionar a implantação de um sistema de gestão organizacional confiável, com propósito de entregar aos clientes serviços e bens conforme as especificações definidas ${ }^{3}$.

As organizações do setor público não estão alheias a esse fenômeno e realizam esforços no sentido da implementação de SGQ4. No Brasil, como em outros países, a aplicabilidade dos preceitos da ISO 9001 para organizações públicas foi impulsionada com a publicação de uma norma específica: a ABNT NBR ISO 18091:2014 - SGQ - Diretrizes para a aplicação da ABNT NBR ISO 9001:2008 em prefeituras ${ }^{5}$, cujo objetivo é estabelecer requisitos de gestão para a obtenção de resultados confiáveis. As organizações na área da saúde também vêm acumulando experiências na institucionalização de tais práticas de SGQ, especialmente as organizações assistenciais, tendo em vista a constante necessidade de garantir, manter e aprimorar a qualidade e a segurança na prestação de serviços de saúde. No âmbito da regulação sanitária de produtos e serviços, o movimento mundial encabeçado pela Organização Mundial da Saúde (OMS) vem discutindo a importância da implantação de SGQ nas Autoridades Reguladoras Nacionais (ARN), visando facilitar a convergência regulatória, a confiança mútua e os mecanismos de reconhecimento entre os Estados-membros ${ }^{6,7}$.

Nesse sentido, a OMS publicou, em 2020, um documento de diretrizes para implantação de SGQ em $\mathrm{ARN}^{7}$, no qual aponta que sistemas regulatórios eficazes são um componente essencial do sistema de saúde e que todos os Estados-membros deveriam ter sistemas regulatórios que: (i) abordem riscos associados ao contexto e oportunidades de melhoria contínua; (ii) demonstrem conformidade com requisitos específicos do SGQ; (iii) garantam qualidade, segurança e eficácia de produtos médicos; e, por fim, (iv) garantam produtos médicos e outras tecnologias de saúde no mercado para atender aos requisitos de seu cliente/cidadão. A OMS destaca também que um SGQ tem potencial para garantir que produtos e serviços sujeitos aos atos normativos de uma agência reguladora cumpram consistentemente padrões estatutários e regulatórios, atendendo, assim, às expectativas de seus clientes/cidadãos.

No Brasil, a Lei Orgânica do Sistema Único de Saúde (SUS), Lei n 8.080, de 19 de setembro de $1990^{8}$, define a vigilância sanitária como um conjunto de ações capazes de eliminar, diminuir ou prevenir riscos à saúde e de intervir nos problemas sanitários decorrentes do meio ambiente, da produção, da circulação de bens e da prestação de serviços de interesse da saúde. A ação da vigilância sanitária no âmbito dos estados, Distrito Federal e municípios tem se mostrado difusa ao longo do tempo, considerando as diferenças de regulamentos, as baixas harmonização e uniformidade de execução das ações e as diferenças na definição de processos globalizados de produção e comercialização de produtos sujeitos à vigilância sanitária. Adicionalmente, a necessidade de ampliação da oferta de produtos brasileiros no exterior mostra a necessidade de reconhecimento da equivalência sanitária por parte das autoridades sanitárias internacionais9.

Qualificar as ações de vigilância sanitária com a incorporação de instrumentos que contribuam para o aprimoramento de processos de gestão tem sido pauta de trabalho da Agência Nacional de Vigilância Sanitária (Anvisa) e dos demais entes do Sistema Nacional de Vigilância Sanitária (SNVS) nos últimos anos. Tornou-se assim agenda importante para o SNVS qualificar as ações de vigilância sanitária com a incorporação de instrumentos que contribuam para o aprimoramento dos processos de gestão, mais especificamente no que diz respeito à $\mathrm{GQ}^{4}$.

$\mathrm{Na}$ busca pelo aprimoramento de práticas nacionais, num contexto de parceria interfederativa, a Resolução da Diretoria Colegiada (RDC) $\mathrm{n}^{\circ} 207$, de 3 de janeiro de $2018^{10}$, da Anvisa, dispõe sobre a organização das ações de vigilância sanitária e regulamenta a necessidade de implementação de um SGQ como condição para que os serviços de Vigilância Sanitária de estados, Distrito Federal e municípios possam assumir responsabilidades por ações de inspeção em indústrias de medicamentos, de insumos farmacêuticos ativos (IFA) e de produtos para saúde das 
classes de risco III - alto risco ao indivíduo e/ou médio risco à saúde pública - e IV - alto risco ao indivíduo e alto risco à saúde pública ${ }^{11,12}$. Nesse contexto, este artigo teve como objetivo identificar a aplicação de práticas de gestão da qualidade, por meio da autoavaliação, em órgãos de Vigilância Sanitária.

\section{MÉTODO}

Esse estudo foi desenvolvido no âmbito do projeto Qualificação da Gestão das Ações Estratégicas de Vigilância Sanitária no SNVS - IntegraVisa II, parceria da Anvisa com o Hospital Alemão Oswaldo Cruz (HAOC), por meio do Programa de Apoio ao Desenvolvimento Institucional do SUS (Proadi-SUS). Trata-se de um estudo exploratório e descritivo, referente à aplicação de práticas de GQ em órgão de Vigilância Sanitária de sete estados e 32 municípios selecionados. Esse estudo foi conduzido em três etapas: (i) levantamento bibliográfico; (ii) pesquisa de campo; e (iii) análise dos dados e discussão de resultados.

O levantamento bibliográfico (i) possibilitou a constituição de referenciais teóricos que fundamentaram a elaboração das discussões de resultados. Foi realizado por meio de buscas em bases bibliográficas relacionadas ao tema, com termos validados conforme os Descritores em Ciências da Saúde (DeCS) ${ }^{13}$, relacionados aos três conjuntos de termos-chave: (a) sistema de gestão da qualidade, e/ou gestão da qualidade, e/ou gestão da qualidade em Saúde, e/ou qualidade; (b) agência reguladora, e/ou vigilância sanitária, e/ou serviço de saúde, e/ou área da saúde, e/ou saúde; e (c) serviço público; e/ou setor público; e/ou governo.

Para a realização da pesquisa de campo (ii), segunda etapa do estudo, estabeleceu-se como recorte a ser estudado, órgãos de
Vigilância Sanitária municipais e estaduais em cujos territórios de atuação existissem, no ano de 2018, pelo menos três unidades industriais de fabricantes de medicamentos e/ou insumos farmacêuticos ativos e/ou produtos para saúde classes de risco III e IV. Foram identificados como integrantes do universo desse estudo os órgãos de Vigilância Sanitária de sete estados - Goiás, Minas Gerais, Paraná, Rio de Janeiro, Rio Grande do Sul, Santa Catarina, São Paulo - e 32 municípios - Amparo, Anápolis, Aparecida de Goiânia, Barueri, Bauru, Belo Horizonte, Campinas, Contagem, Cotia, Curitiba, Diadema, Florianópolis, Goiânia, Guarulhos, Hortolândia, Indaiatuba, Juiz de Fora, Lagoa Santa, Maringá, Mogi Mirim, Nova Lima, Pinhais, Porto Alegre, Ribeirão Preto, Rio Claro, Rio de Janeiro, São Carlos, São José do Rio Preto, São José dos Campos, São Paulo, Sorocaba e Taboão da Serra.

Para a etapa de coleta de dados do estudo, optou-se pela utilização de um formulário eletrônico estruturado, com questões relacionadas aos requisitos iniciais para a implementação de um modelo de SGQ em órgãos de Vigilância Sanitária, baseado na norma ABNT NBR ISO 9001:2015. A referida norma foi considerada adequada e aderente aos entes do SNVS que buscam implantar um SGQ, sendo que o modelo proposto se compõe das seguintes seções: contexto externo/interno, liderança, planejamento, apoio, operações, avaliação de desempenho e melhoria, totalizando sete seções (Figura 1). 0 formulário eletrônico foi enviado aos gestores das 39 Vigilâncias Sanitárias, universo do estudo, acompanhado de um glossário com definições dos principais termos adotados (Quadro 1).

As questões disponibilizadas no formulário eletrônico foram agrupadas conforme as sete seções do modelo proposto, em cada uma das quais foram apresentados os requisitos considerados

MODELO DE SISTEMA DE GESTÃO DA QUALIDADE (SGQ) PARA UNIDADES DO SISTEMA NACIONAL DE VIGILÂNCIA SANITÁRIA

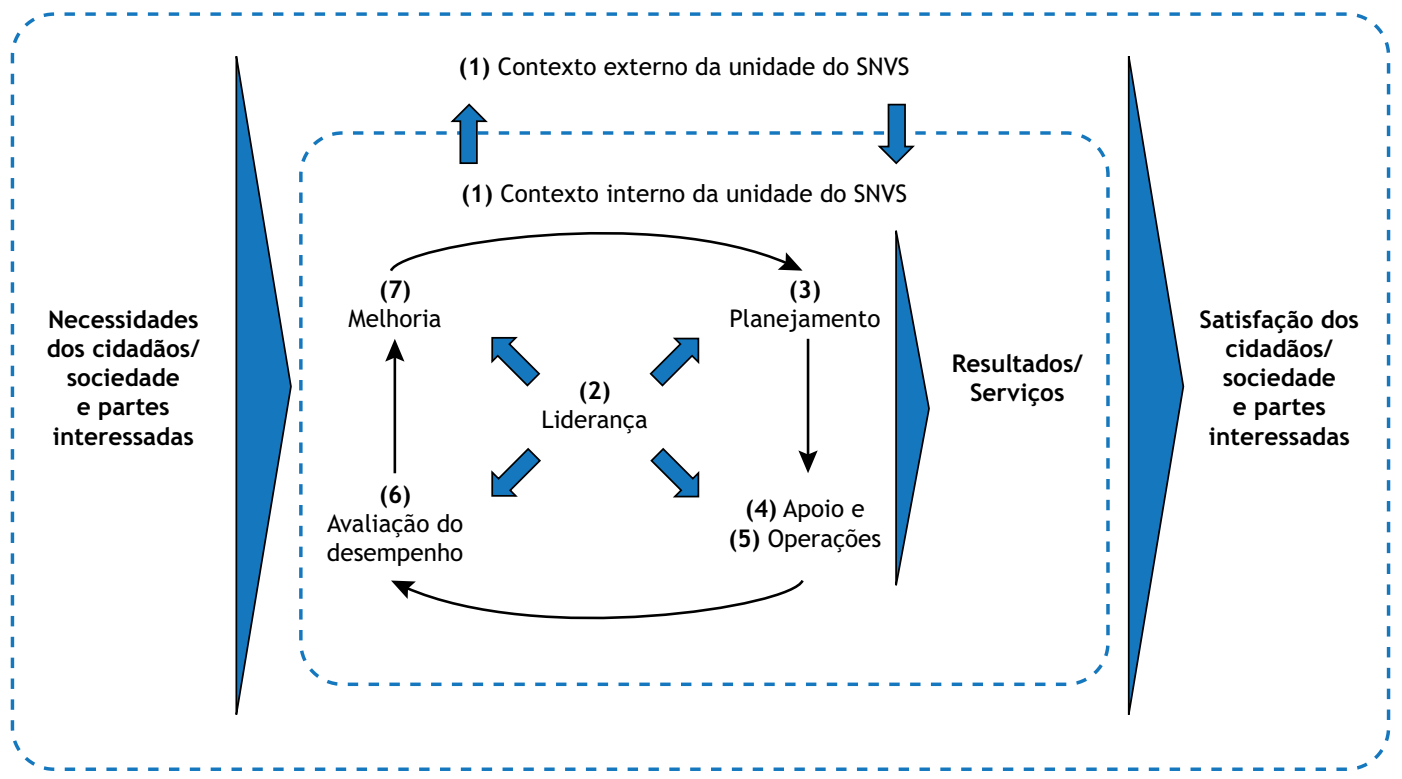

Fonte: Adaptação própria dos autores a partir da referência da norma ABNT NBR ISO 9001:2015, elaborada no âmbito do projeto IntegraVisa²1.

Figura 1. Modelo de Sistema de Gestão da Qualidade para unidades do Sistema Nacional de Vigilância Sanitária, 2019, elaborado com base na norma ABNT NBR ISO 9001:2015. 
como mínimos ou fundamentais para identificar que um órgão de Vigilância Sanitária possua SGQ implantado ou em implantação.

Para apoiar as respostas às questões do formulário eletrônico, foi elaborada uma orientação de preenchimento, na qual os profissionais dos órgãos de Vigilância Sanitária selecionados foram instruídos a responder em equipe, possibilitando, assim, a elaboração de respostas mais completas e mais bem ancoradas nos contextos locais desses órgãos. Também foi disponibilizado um canal direto de comunicação com a equipe que conduziu o estudo, com o objetivo de esclarecer dúvidas que não houvessem sido previamente identificadas. Como complemento às respostas, foi solicitado aos participantes do estudo o envio de evidências - como documentos, modelos, padrões de trabalho, práticas, rotinas, sistemas, manuais ou normativas - que permitissem comprovar objetivamente o cumprimento dos requisitos.

Para identificar a existência ou a execução de práticas relacionadas aos requisitos iniciais das seções na rotina de cada órgão selecionado, solicitou-se que os gestores realizassem um exercício de autoavaliação sobre o atendimento a esses requisitos, considerando a gestão da Vigilância Sanitária de modo global, sob a ótica de duas dimensões: se o requisito inicial é cumprido e se a prática para cumprimento desse requisito é executada apenas em áreas específicas ou na totalidade da organização. No formulário eletrônico, foi incluída uma régua, cuja estratégia foi representar uma medição dessas duas dimensões, de forma gráfica, clara e transparente, permitindo a identificação não somente do quantitativo de práticas relacionadas aos requisitos iniciais atendidos, mas também da abrangência de realização dessas práticas no âmbito do órgão de Vigilância Sanitária (Quadro 2). 0 formulário eletrônico ficou disponível na internet entre as datas de 14 de março e 29 de abril de 2019.

Nos resultados apresentados, não há identificação dos órgãos de Vigilância Sanitária que responderam ao formulário. Para a apresentação dos resultados nesse estudo, os órgãos foram identificados com o código Visa X.YY, onde $X$ indica o estado e $Y Y$ o município. Quando a identificação for Visa X.00, indica que a informação é da Vigilância Sanitária estadual. Os resultados foram estruturados em tabelas e analisados conforme os resultados identificados na pesquisa bibliográfica.

\section{RESULTADOS E DISCUSSÃO}

Do total de órgãos de Vigilância Sanitária selecionados para o estudo (39), no período estabelecido para a coleta de dados, foram recebidas 26 respostas, sendo seis estados e 20 municípios ou $66,7 \%$ do universo estudado. No entanto, ao se considerarem separadamente as respostas recebidas de Vigilâncias Sanitárias municipais e estaduais, temos, respectivamente, $62,5 \%$ e $85,7 \%$ de adesão à pesquisa. Nesse sentido, e levando em conta que os estados são responsáveis por coordenar e dar suporte aos municípios, no seu limite territorial, sobretudo nas ações de vigilância sanitária que envolvem objetos com maior grau de risco sanitário intrínseco às atividades e aos produtos, pode-se afirmar que a quantidade de respostas recebidas aponta para a realidade da

Quadro 1. Descrição das seções da norma ABNT NBR ISO 9001:2015 contempladas no formulário eletrônico do levantamento das práticas de gestão da qualidade em órgãos de Vigilância Sanitária.

\begin{tabular}{|c|c|}
\hline Seção & Descrição \\
\hline Contexto organizacional (seção 1) & $\begin{array}{l}\text { Aborda práticas relacionadas ao contexto organizacional, permitindo a identificação de oportunidades e } \\
\text { obstáculos, além de necessidades das partes interessadas. }\end{array}$ \\
\hline Liderança (seção 2) & $\begin{array}{l}\text { Aborda práticas relacionadas aos papéis, responsabilidades e autoridades organizacionais, ao foco no } \\
\text { cliente e à política e à comunicação do SGQ. }\end{array}$ \\
\hline Planejamento (seção 3) & $\begin{array}{l}\text { Aborda práticas relacionadas ao estabelecimento de ferramentas e de processos para caracterizar } \\
\text { problemas e necessidades, com foco em riscos e oportunidades para a gestão. }\end{array}$ \\
\hline Apoio (seção 4) & $\begin{array}{c}\text { Aborda práticas relacionadas à provisão dos recursos, das pessoas, da infraestrutura e das competências } \\
\text { necessários para o desenvolvimento das ações de vigilância sanitária. }\end{array}$ \\
\hline Operação (seção 5) & $\begin{array}{l}\text { Aborda práticas relacionadas às atividades operacionais, assim como de gerenciamento de processos de } \\
\text { trabalho, enfrentamento de não conformidades e avaliação dos serviços terceirizados. }\end{array}$ \\
\hline Avaliação de desempenho (seção 6) & $\begin{array}{l}\text { Aborda práticas relacionadas a monitoramento, medição, análise e avaliação do desempenho e da } \\
\text { satisfação dos cidadãos/sociedade, auditoria interna e análise crítica pela direção da organização. }\end{array}$ \\
\hline Melhoria (seção 7) & $\begin{array}{c}\text { Aborda práticas relacionadas às práticas de melhoria contínua, tais como identificação e tratamento de } \\
\text { não conformidades e ações corretivas. }\end{array}$ \\
\hline
\end{tabular}

Fonte: Adaptado pelos autores a partir da referência da norma ABNT NBR ISO 9001:2015, elaborado no âmbito do projeto IntegraVisa21.

SQG: Sistemas de Gestão da Qualidade.

Quadro 2. Régua de resposta para a identificação da aplicação das práticas de gestão da qualidade em órgãos de Vigilância Sanitária.

\begin{tabular}{|c|c|c|c|c|c|}
\hline $\begin{array}{c}\text { Não atende a } \\
\text { nenhum dos } \\
\text { requisitos da seção }\end{array}$ & $\begin{array}{c}\text { Atende a menos da } \\
\text { metade dos requisitos } \\
\text { da seção }\end{array}$ & $\begin{array}{c}\text { Atende a menos da } \\
\text { metade dos requisitos } \\
\text { da seção }\end{array}$ & $\begin{array}{c}\text { Atende a mais da } \\
\text { metade ou todos os } \\
\text { requisitos da seção }\end{array}$ & $\begin{array}{c}\text { Atende a mais da } \\
\text { metade dos requisitos } \\
\text { da seção }\end{array}$ & $\begin{array}{c}\text { Atende a todos os } \\
\text { requisitos da seção }\end{array}$ \\
\hline- & Em parte da Visa & Em toda a Visa & Em parte da Visa & Em toda a Visa & Em toda a Visa \\
\hline $0 \%$ & $20 \%$ & $40 \%$ & $60 \%$ & $80 \%$ \\
\hline
\end{tabular}

Fonte: Elaborado pelos autores, 2020.

Visa: Vigilância Sanitária. 
implantação de práticas de GQ no SNVS. O estudo, portanto, permitiu a construção de um panorama de atendimento aos requisitos iniciais por seção do SGQ, considerando a avaliação sob a ótica das duas dimensões metodológicas aplicadas, que se referem às práticas relacionadas aos requisitos iniciais atendidos, desenvolvidas em parte ou na totalidade do órgão (Tabela 1).

A Tabela 1 apresenta, em valores percentuais, a distribuição das 26 Vigilâncias Sanitárias que responderam ao formulário da pesquisa, quanto ao nível de atendimento do conjunto de requisitos referente a cada seção do SGQ. Assim, cada linha da tabela representa uma seção, e seus valores totalizam 100,0\%, o que permite visualizar, linha a linha, qual o percentual de Vigilâncias Sanitárias para cada nível de atendimento dos requisitos, conforme a régua descrita no Quadro 2.

Por um lado, ao se analisarem os resultados da Tabela 1, observa-se que os requisitos relacionados às seções 3 (planejamento) e 4 (apoio) podem ser considerados os conjuntos de requisitos iniciais mais incorporados às práticas dos serviços de Vigilância Sanitária, visto que, somando os três níveis de atendimento mais altos da régua, chega-se, respectivamente, a $61,5 \%$ e $57,7 \%$ das Vigilâncias Sanitárias que responderam ao formulário. Nessas duas seções, portanto, a maior parte dos órgãos de Vigilância Sanitária consultados atendeu, no mínimo, a "mais da metade ou todos os requisitos da seção em parte da Visa", conforme a indicação da régua utilizada.

Por outro lado, tomando como referência os três níveis mais baixos de atendimento da régua, é possível verificar que a maior parte das Vigilâncias Sanitárias participantes da pesquisa atendem a, no máximo, "menos da metade dos requisitos [de cada] seção, em toda a Visa", sendo 53,8\% das Vigilâncias Sanitárias na seção 1 (contexto organizacional); 57,7\%, na 2 (liderança); 65,4\%, na 5 (operações); 80,8\%, na 6 (análise do desempenho); e 65,4\%, na
7 (melhoria). Destacam-se, nesse sentido, os índices superiores a 60,0\% das Vigilâncias Sanitárias nas seções 5, 6 e 7. No caso da seção 5 (operações), o valor pode ser explicado pelo número mais elevado de requisitos em relação às demais seções, ainda que $50,0 \%$ das Vigilâncias Sanitárias tenham respondido que atendem "a menos da metade dos requisitos da seção, em toda a Visa", já que se trata de requisitos ligados aos processos finalísticos, mais comumente executados por elas. No caso das seções 6 e 7, por sua vez, os percentuais devem ser explicados pela incipiente aplicação de processos e práticas de monitoramento e avaliação e de melhoria contínua na gestão das Vigilâncias Sanitárias, destacando-se sobretudo a seção 6 , com $80,8 \%$ delas se concentrando nos três níveis mais baixos da régua.

Vale ressaltar que o acompanhamento da existência de evidências no SGQ tem como propósito a confirmação do atendimento aos requisitos por meio de verificação no momento de auditoria do modelo de gestão. Assim, como relatado anteriormente, foi facultada aos gestores das Vigilâncias Sanitárias participantes do estudo a possibilidade de apresentar documentos que evidenciassem as respostas enviadas sobre o atendimento aos requisitos mínimos. Considerando que o formulário eletrônico era composto por 27 questões, e que 26 Vigilâncias Sanitárias enviaram suas respostas, o limite máximo possível de evidências a serem recebidas era de 702, ressaltando ainda que uma evidência pode atender a mais de um requisito, sendo que apenas 109 foram enviadas pelas Vigilâncias Sanitárias, ou 15,5\% do total possível. Ademais, cabe ressaltar que, dos 26 órgãos que responderam ao formulário eletrônico, um total de nove Vigilâncias Sanitárias não enviou nenhum documento com evidência para suas respostas. Os grupos de evidências foram categorizados de acordo com sua natureza, a fim de analisar a pertinência de serem usados para demonstrar o atendimento aos requisitos mínimos de cada seção do SGQ. Nesse sentido, foram identificadas sete

Tabela 1. Distribuição percentual dos órgãos de Vigilância Sanitária $(n=26)$, segundo o cumprimento de práticas das seções de 1 a 7 , relacionadas a requisitos do Sistema de Gestão da Qualidade, 2019.

\begin{tabular}{|c|c|c|c|c|c|c|}
\hline $\begin{array}{l}\text { Régua } \\
\text { Seção modelo }\end{array}$ & $\begin{array}{l}\text { Não atende a } \\
\text { nenhum dos } \\
\text { requisitos da } \\
\text { seção }\end{array}$ & $\begin{array}{l}\text { Atende a menos } \\
\text { da metade dos } \\
\text { requisitos da seção, } \\
\text { em parte da Visa }\end{array}$ & $\begin{array}{l}\text { Atende a menos } \\
\text { da metade dos } \\
\text { requisitos da seção, } \\
\text { em toda a Visa }\end{array}$ & $\begin{array}{l}\text { Atende a mais da } \\
\text { metade ou todos os } \\
\text { requisitos da seção, } \\
\text { em parte da Visa }\end{array}$ & $\begin{array}{l}\text { Atende a mais } \\
\text { da metade dos } \\
\text { requisitos da seção, } \\
\text { em toda a Visa }\end{array}$ & $\begin{array}{c}\text { Atende a todos } \\
\text { os requisitos da } \\
\text { seção, em toda } \\
\text { a Visa }\end{array}$ \\
\hline $\begin{array}{l}\text { Contexto } \\
\text { organizacional } \\
\text { (seção 1) }\end{array}$ & $15,4 \%$ & $7,7 \%$ & $30,8 \%$ & $19,2 \%$ & $26,9 \%$ & $0,0 \%$ \\
\hline $\begin{array}{l}\text { Liderança } \\
\text { (seção 2) }\end{array}$ & $15,4 \%$ & $15,4 \%$ & $26,9 \%$ & $7,7 \%$ & $19,2 \%$ & $15,4 \%$ \\
\hline $\begin{array}{l}\text { Planejamento } \\
\text { (seção 3) }\end{array}$ & $11,5 \%$ & $11,5 \%$ & $15,4 \%$ & $11,5 \%$ & $19,2 \%$ & $30,8 \%$ \\
\hline $\begin{array}{l}\text { Apoio } \\
\text { (seção 4) }\end{array}$ & $11,5 \%$ & $11,5 \%$ & $19,2 \%$ & $15,4 \%$ & $34,6 \%$ & $7,7 \%$ \\
\hline $\begin{array}{l}\text { Operação } \\
\text { (seção 5) }\end{array}$ & $0,0 \%$ & $15,4 \%$ & $50,0 \%$ & $15,4 \%$ & $19,2 \%$ & $0,0 \%$ \\
\hline $\begin{array}{l}\text { Avaliação de } \\
\text { desempenho } \\
\text { (seção 6) }\end{array}$ & $26,9 \%$ & $23,1 \%$ & $30,8 \%$ & $15,4 \%$ & $0,0 \%$ & $3,8 \%$ \\
\hline $\begin{array}{l}\text { Melhoria } \\
\text { (seção 7) }\end{array}$ & $19,2 \%$ & $26,9 \%$ & $19,2 \%$ & $11,5 \%$ & $15,4 \%$ & $7,7 \%$ \\
\hline
\end{tabular}

Fonte: Elaborada pelos autores, 2020.

Visa: Vigilância Sanitária. 
categorias, apresentadas a seguir com as respectivas quantidades totais de evidências recebidas: 55 documentos da qualidade, 29 documentos de gestão em geral, dez atas/relatórios de atividades realizadas, sete imagens extraídas de sistemas de informação, seis reproduções de legislação, um certificado de curso realizado e uma notícia (Tabela 2 ).

Ainda que o percentual de respostas com evidências seja baixo com relação ao total, é possível estabelecer algumas relações ao se agruparem essas evidências. Assim, foram recebidas 49 evidências das Vigilâncias Sanitárias estaduais participantes e 60, das municipais, o que representa número proporcionalmente menor, considerando que a quantidade de municípios participantes foi mais de três vezes superior à de estados. Esse resultado pode estar relacionado ao fato de as Vigilâncias Sanitárias estaduais terem participado mais ativamente do processo de adesão do Brasil ao Pharmaceutical Inspection Co-operation Scheme (PIC/S), cooperação internacional no campo de boas práticas de fabricação entre as autoridades reguladoras e a indústria farmacêutica, o que envolve, entre outras atividades, a necessidade de implantação de SGQ nos órgãos regulatórios. Nessa mesma linha, observa-se que, do total de 55 documentos da qualidade apresentados, 33 foram enviados pelas Vigilâncias Sanitárias estaduais e 22, pelas municipais.

No que diz respeito à distribuição da quantidade de evidências pelas sete seções do modelo de SGQ, ressalta-se que as seções de 1 a 5 foram contempladas com número mais elevado de evidências que as seções 6 e 7, o que converge com os resultados encontrados na Tabela 1, em que as Vigilâncias Sanitárias apresentaram desempenho inferior no atendimento aos requisitos dessas duas últimas seções. Paralelamente, é interessante comparar a quantidade de evidências das seções 1, 2 e 5, que foram de 20, 21 e 22, respectivamente. Ainda que os valores absolutos sejam muito próximos, cabe ressaltar que o peso da seção 5 , no que tange ao número de requisitos, é maior que o das seções 1 e 2 , o que pode denotar um atendimento proporcionalmente mais completo dos requisitos dessas duas seções em relação aos da seção 5. Nesse mesmo sentido, ao se considerarem apenas os documentos da qualidade, foram enviados $13 \mathrm{e}$ 18 , respectivamente para as seções 1 e 2, e 11, para a seção 5 .

Um resultado importante a ser discutido é o das práticas relacionadas à liderança (seção 2), considerando que cerca de 59,0\% das Vigilâncias Sanitárias se posicionaram nos três níveis mais baixos da régua, isso significa que, no máximo, atendem a menos da metade dos requisitos da seção, em toda a Vigilância Sanitária. Tal resultado pode se caracterizar como uma dificuldade futura, tendo em vista que, segundo Campos $^{14}$, Santos ${ }^{15}$, Carvalho ${ }^{16}$ e Maekawa ${ }^{17}$, o envolvimento da alta direção é fator determinante para o sucesso da implantação de um SGQ nas organizações. Os líderes nas organizações são os responsáveis por dar o direcionamento estratégico e criar condições favoráveis para que as pessoas se engajem no alcance dos objetivos da qualidade. A capacidade do líder e o seu envolvimento na condução do processo de mudança para implantação do SGQ são fatores decisivos para o alcance dos objetivos.

Em estudo realizado com unidades de Laboratórios Centrais de Saúde Pública (LACEN), Campos ${ }^{14}$ afirmou que

como ponto comum entre os LACEN mais desenvolvidos na implantação do sistema da qualidade observa-se o papel fundamental do envolvimento da direção. Aquelas que compreenderam a importância e vislumbraram esta meta como primordial para o fortalecimento e crescimento do laboratório conseguiram avançar e estimular o corpo técnico a se tornar proativo na busca pela qualidade.

Estudo mais recente identifica que o atendimento aos requisitos de liderança foi fundamental para a melhoria da qualidade dos serviços prestados pelos laboratórios de análises clínicas do estado de Santa Catarina ${ }^{18}$.

$\mathrm{Na}$ análise dos resultados por unidade de Vigilância Sanitária, foi possível testar e confirmar a pertinência da régua utilizada para mensurar a aplicação de práticas de GQ como indicativo da

Tabela 2. Distribuição quantitativa das evidências apresentadas pelos órgãos de Vigilância Sanitária, com relação a categorias de evidências, seções do modelo de Sistema de Gestão da Qualidade e esfera de governo, 2019.

\begin{tabular}{|c|c|c|c|c|c|c|c|c|}
\hline $\begin{array}{l}\text { Evidências } \\
\text { Seções/ }\end{array}$ & $\begin{array}{l}\text { Documentos } \\
\text { da qualidade }\end{array}$ & $\begin{array}{l}\text { Documentos } \\
\text { da gestão }\end{array}$ & $\begin{array}{l}\text { Atas/ } \\
\text { relatórios }\end{array}$ & Tela sistema & Legislação & Certificados & Notícias & Geral \\
\hline Seção 1 & 13 & 1 & 3 & 0 & 3 & 0 & 0 & 20 \\
\hline Seção 2 & 18 & 1 & 0 & 0 & 2 & 0 & 0 & 21 \\
\hline Seção 3 & 0 & 10 & 3 & 2 & 0 & 0 & 0 & 15 \\
\hline Seção 4 & 4 & 8 & 4 & 0 & 1 & 1 & 0 & 18 \\
\hline Seção 5 & 11 & 5 & 0 & 5 & 0 & 0 & 1 & 22 \\
\hline Seção 6 & 4 & 4 & 0 & 0 & 0 & 0 & 0 & 8 \\
\hline Seção 7 & 5 & 0 & 0 & 0 & 0 & 0 & 0 & 5 \\
\hline Total EE & 33 & 11 & 3 & 1 & 1 & 0 & 0 & 49 \\
\hline Total MM & 22 & 18 & 7 & 6 & 5 & 1 & 1 & 60 \\
\hline Total & 55 & 29 & 10 & 7 & 6 & 1 & 1 & 109 \\
\hline
\end{tabular}

Fonte: Elaborada pelos autores a partir dos documentos enviados pelas Vigilâncias Sanitárias que responderam ao formulário eletrônico, 2020. EE: Estados; MM: Municípios. 
existência de práticas relacionadas aos requisitos iniciais (Quadro 2). A régua apresentada no formulário eletrônico permitiu verificar e expressar, nesse estudo, que a implantação de práticas para atendimento a requisitos do SGQ, mesmo aqueles considerados iniciais, deve se dar em duas dimensões, considerando a implantação das práticas em si e a abrangência dessa implantação dentro do órgão de Vigilância Sanitária. Nesse sentido, por exemplo, mesmo quando o órgão atende a todos os requisitos de determinada seção, se as respectivas práticas não são executadas em todo o órgão, a resposta ficará em, no máximo, $60,0 \%$ do total da régua. Dessa maneira, pretende-se retratar a complexidade das duas dimensões de implantação do SGQ em valores percentuais, com o intuito de comparar as respostas enviadas pelas Vigilâncias Sanitárias participantes do estudo (Tabela 3).

Nesta análise, apresentados os resultados da régua de medida na Tabela 3, é possível identificar que, conforme o tipo de ente federado, existem diferenças quanto ao grau de organização dos órgãos de Vigilância Sanitária que apontam para a existência de SGQ em fase inicial $(40,0 \%)$ ou em ampliação $(60,0 \%)$ em quase todas as respostas das Vigilâncias Sanitárias estaduais. Porém, somente as Visa 2.00 e
4.00 responderam ter práticas relacionadas a mais de $60,0 \%$ de atendimento dos requisitos iniciais em todas as seções analisadas.

No que diz respeito aos municípios, nenhum deles apresentou o atendimento de mais da metade dos requisitos para o conjunto das sete seções, isto é, o atingimento dos três últimos níveis da régua. $\mathrm{Na}$ análise por seção, tal atingimento enquadra-se se num patamar de $5,0 \%$ a $35,0 \%$ do total de municípios, conforme cada seção (Seção 1 - 35,0\%, Seção 2 - 35,0\%, Seção 3 - 45,0\%, Seção 4 - 45,0\%, Seção 5 - 25,0\%, Seção 6 - 5,0\% e Seção 7 - 25,0\%).

Um resultado que se destaca se relaciona aos percentuais elevados de um terço dos órgãos de Vigilância Sanitária do estado 7.00, aqui identificados como 7.02, 7.03, 7.09 e 7.12, por se tratar de municípios de um estado que descentralizou a execução de ações de vigilância sanitária para os municípios existentes em seu território, incluindo entre essas atividades aquelas relacionadas ao controle de produtos e de serviços de alto risco sanitário. Tais resultados parecem refletir o grau de organização necessário para que os entes realizem ações mais complexas em seus respectivos territórios.

Tabela 3. Percentual de atendimento dos requisitos iniciais das seções de 1 a 7 do Sistema de Gestão da Qualidade por órgãos de Vigilância Sanitária $(\mathrm{n}=26), 2019$.

\begin{tabular}{|c|c|c|c|c|c|c|c|}
\hline Visa & Seção 1 & Seção 2 & Seção 3 & Seção 4 & Seção 5 & Seção 6 & Seção 7 \\
\hline Visa 1.00 & $60,0 \%$ & $60,0 \%$ & $60,0 \%$ & $60,0 \%$ & $60,0 \%$ & $20,0 \%$ & $60,0 \%$ \\
\hline Visa 1.01 & $20,0 \%$ & $20,0 \%$ & $20,0 \%$ & $20,0 \%$ & $20,0 \%$ & $20,0 \%$ & $20,0 \%$ \\
\hline Visa 1.02 & $40,0 \%$ & $80,0 \%$ & $40,0 \%$ & $0,0 \%$ & $40,0 \%$ & $0,0 \%$ & $0,0 \%$ \\
\hline Visa 2.00 & $60,0 \%$ & $60,0 \%$ & $60,0 \%$ & $60,0 \%$ & $60,0 \%$ & $60,0 \%$ & $60,0 \%$ \\
\hline Visa 2.01 & $80,0 \%$ & $40,0 \%$ & $100,0 \%$ & $40,0 \%$ & $40,0 \%$ & $40,0 \%$ & $40,0 \%$ \\
\hline Visa 3.00 & $60,0 \%$ & $40,0 \%$ & $40,0 \%$ & $80,0 \%$ & $40,0 \%$ & $60,0 \%$ & $60,0 \%$ \\
\hline Visa 3.01 & $80,0 \%$ & $80,0 \%$ & $80,0 \%$ & $80,0 \%$ & $40,0 \%$ & $20,0 \%$ & $20,0 \%$ \\
\hline Visa 3.02 & $60,0 \%$ & $20,0 \%$ & $100,0 \%$ & $60,0 \%$ & $40,0 \%$ & $0,0 \%$ & $0,0 \%$ \\
\hline Visa 3.03 & $40,0 \%$ & $100,0 \%$ & $100,0 \%$ & $80,0 \%$ & $40,0 \%$ & $40,0 \%$ & $40,0 \%$ \\
\hline Visa 4.00 & $80,0 \%$ & $100,0 \%$ & $100,0 \%$ & $80,0 \%$ & $80,0 \%$ & $60,0 \%$ & $80,0 \%$ \\
\hline Visa 5.00 & $40,0 \%$ & $100,0 \%$ & $100,0 \%$ & $100,0 \%$ & $60,0 \%$ & $60,0 \%$ & $20,0 \%$ \\
\hline Visa 5.01 & $0,0 \%$ & $0,0 \%$ & $0,0 \%$ & $0,0 \%$ & $20,0 \%$ & $0,0 \%$ & $20,0 \%$ \\
\hline Visa 6.00 & $60,0 \%$ & $40,0 \%$ & $80,0 \%$ & $80,0 \%$ & $40,0 \%$ & $0,0 \%$ & $20,0 \%$ \\
\hline Visa 6.01 & $80,0 \%$ & $40,0 \%$ & $100,0 \%$ & $80,0 \%$ & $80,0 \%$ & $40,0 \%$ & $80,0 \%$ \\
\hline Visa 7.01 & $40,0 \%$ & $20,0 \%$ & $20,0 \%$ & $20,0 \%$ & $20,0 \%$ & $20,0 \%$ & $80,0 \%$ \\
\hline Visa 7.02 & $40,0 \%$ & $80,0 \%$ & $80,0 \%$ & $80,0 \%$ & $60,0 \%$ & $20,0 \%$ & $40,0 \%$ \\
\hline Visa 7.03 & $40,0 \%$ & $100,0 \%$ & $100,0 \%$ & $100,0 \%$ & $80,0 \%$ & $100,0 \%$ & $100,0 \%$ \\
\hline Visa 7.04 & $20,0 \%$ & $20,0 \%$ & $20,0 \%$ & $20,0 \%$ & $20,0 \%$ & $20,0 \%$ & $20,0 \%$ \\
\hline Visa 7.05 & $80,0 \%$ & $80,0 \%$ & $60,0 \%$ & $60,0 \%$ & $40,0 \%$ & $0,0 \%$ & $20,0 \%$ \\
\hline Visa 7.06 & $40,0 \%$ & $40,0 \%$ & $40,0 \%$ & $40,0 \%$ & $40,0 \%$ & $40,0 \%$ & $40,0 \%$ \\
\hline Visa 7.07 & $0,0 \%$ & $0,0 \%$ & $80,0 \%$ & $40,0 \%$ & $40,0 \%$ & $0,0 \%$ & $0,0 \%$ \\
\hline Visa 7.08 & $0,0 \%$ & $0,0 \%$ & $0,0 \%$ & $40,0 \%$ & $40,0 \%$ & $40,0 \%$ & $0,0 \%$ \\
\hline Visa 7.09 & $80,0 \%$ & $40,0 \%$ & $100,0 \%$ & $80,0 \%$ & $80,0 \%$ & $40,0 \%$ & $100,0 \%$ \\
\hline Visa 7.10 & $40,0 \%$ & $40,0 \%$ & $40,0 \%$ & $40,0 \%$ & $40,0 \%$ & $40,0 \%$ & $40,0 \%$ \\
\hline Visa 7.11 & $0,0 \%$ & $0,0 \%$ & $0,0 \%$ & $0,0 \%$ & $40,0 \%$ & $0,0 \%$ & $0,0 \%$ \\
\hline Visa 7.12 & $80,0 \%$ & $80,0 \%$ & $80,0 \%$ & $80,0 \%$ & $80,0 \%$ & $40,0 \%$ & $80,0 \%$ \\
\hline
\end{tabular}

Fonte: Eleaborada pelos autores, 2020.

Visa: Vigilância Sanitária. 
No Brasil, as singularidades do modelo federativo e da conformação da área da vigilância sanitária são apontadas como pano de fundo de um esforço de coordenação federativa que visa à construção do SUS. A vigilância sanitária contribui tanto para a concretização do direito social à saúde, quanto para a interferência no funcionamento do mercado a ela sujeito, conferindo maiores previsibilidade, transparência e estabilidade ao processo e à atuação regulatórios, a fim de propiciar um ambiente seguro à população e favorável ao desenvolvimento social e econômico do país ${ }^{19,20}$. No entanto, segundo Lucchese ${ }^{4}$, a pobreza do debate sobre o SNVS evidencia as dificuldades que se têm apresentado no processo de estruturação do próprio SNVS, sobretudo aqueles referentes à definição de papéis dos entes de cada nível de governo e ao processo de descentralização e coordenação federativa ${ }^{4}$.

As diferenças existentes na organização dos órgãos de Vigilância Sanitária, identificadas conforme o tipo de ente, considerando também a forma de organização regional, repercutem diretamente na construção do SUS e no alcance dos direitos à saúde. 0 enfoque normativo da Vigilância Sanitária, configurado por uma série de rotinas que visam o controle quantitativo de metas relacionadas à cobertura das ações, pode favorecer a inovação por meio da implantação de um modelo de gestão que agregue aspectos relativos à qualidade das ações executadas e ao benefício social.

\section{CONCLUSÕES}

Os resultados obtidos neste estudo contribuem para a compreensão do nível de adequação e do respectivo esforço que os entes que compõem o SNVS deverão empreender para ajustar-se às exigências de melhoria de gestão impostas pelo ambiente regulatório nacional e internacional. Conhecer em que medida há iniciativas de SGQ no SNVS e traduzir o que precisa ser feito para o avanço de iniciativas de melhoria de processos de trabalho e de gestão são etapas fundamentais para alcançar aderência e viabilidade na implantação de um modelo de SGQ.

Foi possível traçar um panorama do atendimento aos requisitos iniciais relacionados às sete seções do modelo de SGQ para unidades do SNVS. Neste panorama, constatou-se que algumas práticas de gestão da qualidade já fazem parte dos processos de trabalho de todos os órgãos de Vigilância Sanitária que participaram do estudo. As práticas mais disseminadas estão relacionadas às seções de planejamento e apoio.

Possivelmente, a longa tradição de consolidação das práticas de planejamento no âmbito do SUS e a forte estruturação normativo burocrática relacionada às atividades de apoio podem ter contribuído para que requisitos ligados a estas seções estejam mais aplicados. Porém, na medida em que se ampliam as práticas relacionadas aos requisitos das seções de avaliação de desempenho (seção 6) e melhorias (seção 7), tornam-se maiores os desafios a serem considerados quando da implementação de um modelo de SGQ no SNVS. Os achados relacionados à baixa aplicação dos requisitos destas seções podem indicar que os modelos atuais de gestão destes serviços seguem padrões normativos burocráticos e encontram-se estagnados, sem uma dinâmica de avaliação dos resultados e melhoria a partir deles, duas diretrizes fundamentais do SGQ, com reflexos diretos na satisfação das necessidades de cidadãos e demais partes interessadas.

Como limitação deste estudo, é possível identificar o fato de que o material coletado - objeto de análise - é resultante de autoavaliação por parte das equipes dos órgãos de Vigilância Sanitária, sendo que um quantitativo baixo de evidências foi apresentado comparado ao limite superior possível, calculado considerando o total de requisitos analisados. Não foi previsto utilizar técnicas de verificação das informações obtidas para comparação com a realidade dos fatos, o que permitiria sanar impressões e corrigir possíveis erros de interpretação das práticas e das evidências sob análise, inclusive no que se refere à ausência de familiaridade dos profissionais com os termos utilizados na linguagem da GQ. Também, a seleção dos entes para compor o estudo não se deu com o objetivo de se ter uma representatividade da realidade do conjunto do SNVS, mas sim de conhecer as práticas já implantadas em órgãos de Vigilância Sanitária que possuem, sob a sua abrangência de atuação, um parque industrial de empresas fabricantes de medicamentos, IFA e produtos para saúde classes de risco III e IV, portanto estabelecimentos com atividades e produtos de alto risco sanitário. A qualificação da ação das Vigilâncias Sanitárias nesses territórios contribui para o cumprimento das exigências dos processos globalizados de produção e comercialização de produtos, visando a ampliar o mercado de produtos brasileiros no exterior a partir do atendimento aos padrões de convergência regulatória internacional.

Por fim, os resultados desse estudo sinalizaram incipiência na aplicação dos requisitos iniciais por parte dos órgãos analisados, indicando o potencial e o amplo espaço para implementação de princípios e diretrizes da GQ, a partir do desenvolvimento de estratégias e instrumentos, compartilhados e contínuos, para a execução de planejamento, monitoramento, avaliação e auditoria, por órgãos de Vigilância Sanitária nas três esferas de governo, com possível aumento da efetividade das ações de promoção e proteção à saúde, apoiando a transformação das práticas no SNVS e contribuindo para a disponibilização, com segurança e qualidade, de produtos e serviços regulados.

\section{REFERÊNCIAS}

1. Monaco FF, Mello AFM. A gestão da qualidade total e a reestruturação industrial e produtiva: um breve resgate histórico. Race Rev Adm Contab Econ. 2007;6(1):7-26.

2. Carpinetti LCR, Miguel PAC, Gerolamo MC. Gestão da qualidade ISO 9001:2000: princípios e requisitos. São Paulo: Atlas; 2009.
3. Associação Brasileira de Normas Técnicas - ABNT. ISO 9001:2015: sistemas de gestão da qualidade: requisitos. Rio de Janeiro: Associação Brasileira de Normas Técnicas; 2015.

4. Lucchese G. Globalização e regulação sanitária: os rumos da vigilância sanitária no Brasil [tese]. Rio de Janeiro: Fundação Oswaldo Cruz; 2001. 
5. Associação Brasileira de Normas Técnicas - ABNT. ISO 18091:2014: sistemas de gestão da qualidade: diretrizes para a aplicação da ABNT NBR ISO 9001:2008 em prefeituras. Rio de Janeiro: Associação Brasileira de Normas Técnicas; 2014.

6. World Health Organization - WHO. Delivering quality health services: a global imperative for universal health coverage. Geneva: World Health Organization; 2018[acesso 30 ago 2019]. Disponível em: https://apps.who.int/iris/bitstream/ handle /10665/272465/9789241513906-eng.pdf

7. World Health Organization - WHO. Annex 13: WHO guideline on the implementation of quality management systems for national regulatory authorities. Geneva: World Health Organization; 2020[acesso 5 fev 2021]. Disponível em: https://www.who. int/docs/default-source/medicines/norms-and-standards/ guidelines/trs1025/trs1025-annex13.pdf?sfvrsn=8e6a17ee_2

8. Brasil. Lei $N^{\circ} 8.080$, de 19 de setembro de 1990. Dispõe sobre as condições para a promoção, proteção e recuperação da saúde, a organização e o funcionamento dos serviços correspondentes e dá outras providências. Diário Oficial União. 20 set 1990.

9. Alencar MLSM, Bacelar VMB, Magajewski F, Silva WM, Sousa AIA. Qualificação das ações de vigilância sanitária: harmonização e descentralização. Vigil Sanit Debate. 2019;7(4):111-18. https://doi.org/10.22239/2317-269x.01401

10. Agência Nacional de Vigilância Sanitária - Anvisa. Resolução RDC $N^{\circ}$ 207, de 3 de janeiro de 2018. Dispõe sobre a organização das ações de vigilância sanitária, exercidas pela união, estados, Distrito Federal e municípios, relativas à autorização de funcionamento, licenciamento, registro, certificação de boas práticas, fiscalização, inspeção e normatização, no âmbito do sistema nacional de vigilância sanitária SNVS. Diário Oficial União. 5 jan 2018.

11. Agência Nacional de Vigilância Sanitária - Anvisa. Resolução RDC N 61, de 18 de novembro de 2011. Dispõe sobre as regras de classificação dos produtos para diagnóstico de uso in vitro e dá outras providências. Diário Oficial União. 19 nov 2011.

12. Agência Nacional de Vigilância Sanitária - Anvisa. Resolução RDC No 153, de 26 de abril de 2017. Dispõe sobre a classificação do grau de risco para as atividades econômicas sujeitas à vigilância sanitária, para fins de licenciamento, e dá outras providências. Diário Oficial União. 27 abr 2017.
13. Centro Latino-Americano e do Caribe de Informação em Ciências da Saúde - Bireme. Biblioteca virtual em saúde: descritores em ciências da saúde. São Paulo: Centro LatinoAmericano e do Caribe de Informação em Ciências da Saúde; 2020[acesso 26 fev 2020]. Disponível em: http://decs.bvs.br/

14. Campos A, Mattos S. Avaliação de requisitos referentes à implantação do sistema de gestão da qualidade nos laboratórios de saúde pública. Rev Inst Adolfo Lutz. 2009;68(3):461-70.

15. Santos LL, Mainier FB. Fatores críticos para implantação do sistema de gestão da qualidade em laboratórios de ensaio e calibração. In: Anais do $7^{\circ}$ Congresso Nacional de Excelência em Gestão; Rio de Janeiro, Brasil. Rio de Janeiro: Congresso Nacional de Excelência em Gestão; 2011.

16. Carvalho R. Implantação de sistema de gestão da qualidade: um estudo de caso em uma importadora de medicamentos. In: Anais do $7^{\circ}$ Congresso Nacional de Excelência em Gestão; Rio de Janeiro, Brasil. Rio de Janeiro: Congresso Nacional de Excelência em Gestão; 2011.

17. Maekawa R, Carvalho MM, Oliveira OJ. Um estudo sobre a certificação ISO 9001 no Brasil: mapeamento de motivações, benefícios e dificuldades. Gest Prod. 2013;20(4):763-79. https://doi.org/10.1590/S0104-530X2013005000003

18. Lescowicz G, Melo R, Rateke E, Martinello F. Dez anos da RDC 302/2005: avaliação da implantação em laboratórios de análise clínicas do estado de Santa Catarina. Rev Bras Anal Clin. 2018;50(2):161-70. https://doi.org/10.21877/2448-3877.201800617

19. Brasil. Lei $N^{\circ} 9.782$, de 26 de janeiro de 1999. Define o Sistema Nacional de Vigilância Sanitária, cria a Agência Nacional de Vigilância Sanitária, e dá outras providências. Diário Oficial União. 27 jan 1999.

20. Agência Nacional de Vigilância Sanitária - Anvisa. Cartilha de vigilância sanitária. 2a ed. Brasília: Agência Nacional de Vigilância Sanitária; 2002.

21. Agência Nacional de Vigilância Sanitária - Anvisa. Projeto qualificação da gestão das ações estratégicas de vigilância sanitária no sistema nacional de vigilância sanitária SNVS: IntegraVisa II. Brasília: Agência Nacional de Vigilância Sanitária; 2018.

\section{Agradecimentos}

Agradecemos ao Hospital Alemão Oswaldo Cruz (HAOC), que, por meio do Programa de Apoio ao Desenvolvimento Institucional do Sistema Único de Saúde (Proadi-SUS), financiou o estudo. Agradecemos também a todos os participantes do projeto IntegraVisa II, profissionais da Anvisa, consultores e servidores do HAOC, que possibilitaram a realização desse trabalho.

\section{Contribuição dos Autores}

Silva WM, Bacelar VMB, Sousa AIA, Barca DAAV, Santos CM - Concepção, planejamento (desenho do estudo), análise, interpretação dos dados e redação do trabalho. Zanetta BL - Concepção, planejamento (desenho do estudo), aquisição, análise, interpretação dos dados e redação do trabalho. Todos os autores aprovaram a versão final do trabalho.

Os autores informam não haver qualquer potencial conflito de interesse com pares e instituições, políticos ou financeiros deste estudo.

Licença CC BY-NC atribuição não comercial. Com essa licença é permitido acessar, baixar (download), copiar, imprimir, compartilhar, reutilizar e distribuir os artigos, desde que para uso não comercial e com a citação da fonte, conferindo os devidos créditos de autoria e menção à Visa em Debate. Nesses casos, nenhuma permissão é necessária por parte dos autores ou dos editores. 